\title{
SEMI-INFINITE COHOMOLOGY AND SUPERCONFORMAL ALGEBRAS
}

\author{
By Elena Poletaeva
}

\begin{abstract}
We describe representations of certain superconformal algebras in the semi-infinite Weil complex related to the loop algebra of a complex finite-dimensional Lie algebra and in the semi-infinite cohomology. We show that in the case where the Lie algebra is endowed with a nondegenerate invariant symmetric bilinear form, the relative semi-infinite cohomology of the loop algebra has a structure, which is analogous to the classical structure of the de Rham cohomology in Kähler geometry.
\end{abstract}

\section{COHOMOLOGIE SEMI-INFINIE ET ALGÈBRES SUPERCONFORMES}

Résumé - Nous décrivons des représentations de certaines algèbres superconformes dans le complexe de Weil semi-infini de l'algèbre des lacets d'une algèbre de Lie complexe de dimension finie et dans la cohomologie semi-infinie. Nous démontrons que dans le cas où l'algèbre de Lie est munie d'une forme symétrique non dégénérée invariante, la cohomologie semi-infinie relative de l'algèbre des lacets admet une structure, qui est l'analogue de la structure classique de la cohomologie de de Rham des variétés kählériennes.

\section{Introduction}

B. Feigin and E. Frenkel have introduced a semi-infinite analogue of the Weil complex based on the space

$$
W^{\frac{\infty}{2}+*}(\mathfrak{g})=S^{\frac{\infty}{2}+*}(\mathfrak{g}) \otimes \Lambda^{\frac{\infty}{2}+*}(\mathfrak{g})
$$

In their construction $\mathfrak{g}=\oplus_{n \in \mathbb{Z}} \mathfrak{g}_{n}$ is a graded Lie algebra, $S^{\frac{\infty}{2}+*}(\mathfrak{g})$ and $\Lambda^{\frac{\infty}{2}+*}(\mathfrak{g})$ are some semi-infinite analogues of the symmetric and exterior power modules, $[\mathrm{FF}]$. As in the classical case, two differentials, $d$ and $h$, are defined on $W^{\frac{\infty}{2}+*}(\mathfrak{g})$. They are analogous to the differential in Lie algebra (co)homology and the Koszul differential, respectively. The semi-infinite Weil complex

$$
\left\{W^{\frac{\infty}{2}+*}(\mathfrak{g}), d+\mathrm{h}\right\}
$$

is acyclic similarly to the classical Weil complex. The cohomology of the complex

$$
\left\{W^{\frac{\infty}{2}+*}(\mathfrak{g}), d\right\}
$$


is called the semi-infinite cohomology of $\mathfrak{g}$ with coefficients in its "adjoint semi-infinite symmetric powers" $H^{\frac{\infty}{2}+*}\left(\mathfrak{g}, S^{\frac{\infty}{2}+*}(\mathfrak{g})\right)$. One can also define the relative semi-infinite Weil complex $W_{\text {rel }}^{\frac{\infty}{2}+*}(\mathfrak{g})$ (relatively $\mathfrak{g}_{0}$ ), and the relative semi-infinite cohomology $H^{\frac{\infty}{2}+*}\left(\mathfrak{g}, \mathfrak{g}_{0}, S^{\frac{\infty}{2}+*}(\mathfrak{g})\right),[\mathrm{FF}]$.

E. Getzler has shown that the semi-infinite Weil complex of the Virasoro algebra admits an action of the $N=2$ superconformal algebra, [G].

Recall that a superconformal algebra (SCA) is a simple complex Lie superalgebra $\mathfrak{s}$, such that it contains the centerless Virasoro algebra (i.e. Witt algebra) Witt $=\oplus_{n \in \mathbb{Z}} \mathbb{C} L_{n}$ as a subalgebra, and has growth 1 . The $\mathbb{Z}$-graded superconformal algebras are ones for which $a d L_{0}$ is diagonalizable with finite-dimensional eigenspaces, [KL]:

$$
\mathfrak{s}=\oplus_{j} \mathfrak{s}_{j}, \mathfrak{s}_{j}=\left\{x \in \mathfrak{s} \mid\left[L_{0}, x\right]=j x\right\}
$$

In this work we consider the semi-infinite Weil complex constructed for the next natural (after the Virasoro algebra) class of graded Lie algebras: the loop algebras of the complex finite-dimensional Lie algebras. The action of the Virasoro algebra on such complex is ensured by the fact that it has a structure of a vertex operator superalgebra (see $[\mathrm{Ak}])$.

Let $\mathfrak{g}$ be a complex finite-dimensional Lie algebra, and $\tilde{\mathfrak{g}}=\mathfrak{g} \otimes \mathbb{C}\left[t, t^{-1}\right]$ be the corresponding loop algebra. We obtain a representation of the $N=2 \mathrm{SCA}$ in the semi-infinite Weil complex $W^{\frac{\infty}{2}+*}(\tilde{\mathfrak{g}})$ and in the semi-infinite cohomology $H^{\frac{\infty}{2}+*}\left(\tilde{\mathfrak{g}}, S^{\frac{\infty}{2}+*}(\tilde{\mathfrak{g}})\right)$ with central charge 3dimg. We extend the representation of the $N=2 \mathrm{SCA}$ in $W^{\frac{\infty}{2}+*}(\tilde{\mathfrak{g}})$ to a representation of the one-parameter family $\hat{S}^{\prime}(2, \alpha)$ of deformations of the $N=4$ SCA (see $[\mathrm{Ad}]$ and $[\mathrm{KL}])$. In the case, when $\mathfrak{g}$ is endowed with a non-degenerate invariant symmetric bilinear form, we obtain a representation of $\hat{S}^{\prime}(2,0)$ in $H^{\frac{\infty}{2}+*}\left(\tilde{\mathfrak{g}}, S^{\frac{\infty}{2}+*}(\tilde{\mathfrak{g}})\right)$. Finally, there exists a representation of a central extension of the Lie superalgebra of all derivations of $S^{\prime}(2,0)$ in the relative semi-infinite cohomology $H^{\frac{\infty}{2}+*}\left(\tilde{\mathfrak{g}}, \tilde{\mathfrak{g}}_{0}, S^{\frac{\infty}{2}+*}(\tilde{\mathfrak{g}})\right)$.

It was shown in [FGZ] that the cohomology of the relative semi-infinite complex $C_{\infty}^{*}\left(\mathfrak{l}, \mathfrak{l}_{0}, V\right)$, where $\mathfrak{l}$ is a complex graded Lie algebra, and $V$ is a graded Hermitian l-module, has (under certain conditions) a structure analogous to that of de Rham cohomology in Kähler geometry.

Recall that given a compact Kähler manifold $M$, there exist a number of classical operators on the space of differential forms on $M$, such as the differentials $\partial, \bar{\partial}, d, d_{c}$, their corresponding adjoint operators and the associated Laplacians (see $[\mathrm{GH}]$ ). There also exists an action of $\mathfrak{s t}(2)$ on $H^{*}(M)$ according to the Lefschetz theorem. All these operators satisfy 
a series of identities known as Hodge identities, [GH]. Naturally, the classical operators form a finite-dimensional Lie superalgebra.

We show that given a complex finite-dimensional Lie algebra $\mathfrak{g}$ endowed with a nondegenerate invariant symmetric bilinear form, there exist the analogues of the classical

operators on the complex $W_{\text {rel }}^{\frac{\infty}{2}+*}(\tilde{\mathfrak{g}})$. We prove that the exterior derivations of $S^{\prime}(2,0)$ form an $\mathfrak{s l}(2)$, and observe that they define an $\mathfrak{s l}(2)$-module structure on $H^{\frac{\infty}{2}+*}\left(\tilde{\mathfrak{g}}, \tilde{\mathfrak{g}}_{0}, S^{\frac{\infty}{2}}+*(\tilde{\mathfrak{g}})\right)$, which is the analogue of the $\mathfrak{s l}(2)$-module structure on the de Rham cohomology in Kähler geometry.

The action of $\hat{S}^{\prime}(2,0)$ provides $H^{\frac{\infty}{2}+*}\left(\tilde{\mathfrak{g}}, \tilde{\mathfrak{g}}_{0}, S^{\frac{\infty}{2}+*}(\tilde{\mathfrak{g}})\right)$ with eight series of quadratic operators. In particular, they include the semi-infinite Koszul differential $h$, and the semiinfinite analogue of the homotopy operator (cf. $[\mathrm{Fu}]$ ). We prove that the degree zero part of the $\mathbb{Z}$-grading of $S^{\prime}(2,0)$ defined by the element $L_{0} \in W i t t$, is isomorphic to the Lie superalgebra of classical operators in Kähler geometry.

It would be interesting to interpret the superconformal algebra $S^{\prime}(2,0)$ as "affinization" of the classical operators in the case of an infinite-dimensional manifold.

This work is partly based on [P1]-[P3].

\section{Semi-infinite Weil complex}

The semi-infinite Weil complex of a graded Lie algebra was introduced by B. Feigin and E. Frenkel in $[\mathrm{FF}]$. Recall the necessary definitions. More generally, let $V=\oplus_{n \in \mathbb{Z}} V_{n}$ be a graded vector space over $\mathbb{C}$, such that $\operatorname{dim} V_{n}<\infty$. Let $V^{\prime}=\oplus_{n \in \mathbb{Z}} V_{n}^{\prime}$ be the restricted dual of $V$. The linear space $V \oplus V^{\prime}$ carries non-degenerate skew-symmetric and symmetric bilinear forms: $(\cdot, \cdot)$ and $\{\cdot, \cdot\}$. Let $H(V)$ and $C(V)$ be the quotients of the tensor algebra $T^{*}\left(V \oplus V^{\prime}\right)$ by the ideals generated by the elements of the form $x y-y x-(x, y)$ and $x y+y x-\{x, y\}$, respectively, where $x, y \in V \oplus V^{\prime}$. We fix $K \in \mathbb{Z}$. Let $V=V_{+} \oplus V_{-}$be the corresponding polarization of $V: V_{+}=\oplus_{n>K} V_{n}, V_{-}=\oplus_{n \leq K} V_{n}$.

The symmetric algebra $S^{*}\left(V_{+} \oplus V_{-}^{\prime}\right)$ is a subalgebra of $H(V)$ and the exterior algebra $\Lambda^{*}\left(V_{+} \oplus V_{-}^{\prime}\right)$ is a subalgebra of $C(V)$. Let $S^{\frac{\infty}{2}+*}(V), \Lambda^{\frac{\infty}{2}+*}(V)$ be the representations of $H(V)$ and $C(V)$ induced from the trivial representations $\left\langle\mathbf{1}_{S}\right\rangle$ and $\left\langle\mathbf{1}_{\Lambda}\right\rangle$ of $S^{*}\left(V_{+} \oplus V_{-}^{\prime}\right)$ and of $\Lambda^{*}\left(V_{+} \oplus V_{-}^{\prime}\right)$, respectively. Thus we obtain some semi-infinite analogues of symmetric and exterior power modules. Denote the actions of $H(V)$ and $C(V)$ on these modules by $\beta(x), \gamma\left(x^{\prime}\right)$ and $\tau(x), \varepsilon\left(x^{\prime}\right)$, respectively, for $x \in V, x^{\prime} \in V^{\prime}$. Notice that each element of $S^{\frac{\infty}{2}+*}(V)$ and of $\Lambda^{\frac{\infty}{2}+*}(V)$ is a finite linear combination of the monomials of 
the type $\gamma\left(x_{1}^{\prime}\right) \ldots \gamma\left(x_{k}^{\prime}\right) \beta\left(y_{1}\right) \ldots \beta\left(y_{m}\right) \mathbf{1}_{S}$ and of the type $\varepsilon\left(x_{1}^{\prime}\right) \ldots \varepsilon\left(x_{k}^{\prime}\right) \tau\left(y_{1}\right) \ldots \tau\left(y_{m}\right) \mathbf{1}_{\Lambda}$, respectively, where $x_{1}^{\prime}, \ldots, x_{k}^{\prime} \in V_{+}^{\prime}, y_{1}, \ldots y_{m} \in V_{-}$. Let $\operatorname{Deg} \varepsilon\left(x^{\prime}\right)=\operatorname{Deg} \gamma\left(x^{\prime}\right)=1$, and $\operatorname{Deg} \tau(x)=\operatorname{Deg} \beta(x)=-1$. Correspondingly, we obtain $\mathbb{Z}$-gradings on the spaces of semi-infinite power modules: $S^{\frac{\infty}{2}+*}(V)=\oplus_{i \in \mathbb{Z}} S^{\frac{\infty}{2}+i}(V), \Lambda^{\frac{\infty}{2}+*}(V)=\oplus_{i \in \mathbb{Z}} \Lambda^{\frac{\infty}{2}+i}(V)$.

Let $\left\{e_{i}\right\}_{i \in \mathbb{Z}}$ be a homogeneous basis of $V$ so that if $i \in \mathbb{Z}$, then $e_{i} \in V_{n}$ for some $n \in \mathbb{Z}$, and if $e_{i} \in V_{n}$, then $e_{i+1} \in V_{n}$ or $e_{i+1} \in V_{n+1}$. Let $\left\{e_{i}^{\prime}\right\}_{i \in \mathbb{Z}}$ be the dual basis. Let $i_{0} \in \mathbb{Z}$ be such that $e_{i_{0}} \in V_{K}$ and $e_{i_{0}+1} \in V_{K+1}$.

Notice that one can think of $\Lambda^{\frac{\infty}{2}+*}(V)$ as the vector space spanned by the elements $w=e_{i_{1}}^{\prime} \wedge e_{i_{2}}^{\prime} \wedge \ldots$ such that there exists $N(w) \in \mathbb{Z}$ such that $i_{n+1}=i_{n}-1$ for $n>N(w)$. Then $\mathbf{1}_{\Lambda}=e_{i_{0}}^{\prime} \wedge e_{i_{0}-1}^{\prime} \wedge \ldots$ is a vacuum vector in this space. The actions of $\varepsilon\left(x^{\prime}\right), \tau(x)$ are, respectively, the exterior multiplication and contraction in the space of semi-infinite exterior products.

Let $\mathfrak{g}=\oplus_{n \in \mathbb{Z}} \mathfrak{g}_{n}$ be a graded Lie algebra over $\mathbb{C}$, such that $\operatorname{dim} \mathfrak{g}_{n}<\infty$. Let $\phi$ be a representation of $\mathfrak{g}$ in $V$ so that

$$
\phi\left(\mathfrak{g}_{n}\right) V_{k} \subset V_{k+n} .
$$

One can define the projective representations $\rho$ and $\pi$ of $\mathfrak{g}$ in $\Lambda^{\frac{\infty}{2}+*}(V)$ and $S^{\frac{\infty}{2}+*}(V)$, respectively:

$$
\begin{aligned}
& \rho(x)=\sum_{i \in \mathbb{Z}}: \tau\left(\phi(x) e_{i}\right) \varepsilon\left(e_{i}^{\prime}\right):, \\
& \pi(x)=\sum_{i \in \mathbb{Z}}: \beta\left(\phi(x) e_{i}\right) \gamma\left(e_{i}^{\prime}\right):,
\end{aligned}
$$

where $x \in \mathfrak{g}$, and where the double colons : : denote a normal ordering operation:

$$
: \tau\left(e_{j}\right) \varepsilon\left(e_{i}^{\prime}\right):=\left\{\begin{array}{r}
\tau\left(e_{j}\right) \varepsilon\left(e_{i}^{\prime}\right) \text { if } i \leq i_{0} \\
-\varepsilon\left(e_{i}^{\prime}\right) \tau\left(e_{j}\right) \text { if } i>i_{0}
\end{array}\right\}, \quad: \beta\left(e_{j}\right) \gamma\left(e_{i}^{\prime}\right):=\left\{\begin{array}{l}
\beta\left(e_{j}\right) \gamma\left(e_{i}^{\prime}\right) \text { if } i \leq i_{0} \\
\gamma\left(e_{i}^{\prime}\right) \beta\left(e_{j}\right) \text { if } i>i_{0}
\end{array}\right\} .
$$

Thus

$$
\rho(x) \mathbf{1}_{\Lambda}=\pi(x) \mathbf{1}_{S}=0 \text { for } x \in \mathfrak{g}_{0}
$$

and

$$
\begin{aligned}
& {[\rho(x), \rho(y)]=\rho([x, y])+c_{\Lambda}(x, y),} \\
& {[\pi(x), \pi(y)]=\pi([x, y])+c_{S}(x, y),}
\end{aligned}
$$

where $x, y \in \mathfrak{g}$ and $c_{\Lambda}, c_{S}$ are 2-cocycles. Notice that $c_{\Lambda}=-c_{S}$. Let

$$
W^{\frac{\infty}{2}+*}(V)=S^{\frac{\infty}{2}+*}(V) \otimes \Lambda^{\frac{\infty}{2}+*}(V) .
$$


Since the cocycles corresponding to the projective representations cancel, the representation $\theta(x)=\rho(x)+\pi(x)$ of $\mathfrak{g}$ in $W^{\frac{\infty}{2}+*}(V)$ is well-defined. We define a $\mathbb{Z}$-grading on $W^{\frac{\infty}{2}+*}(V)$ setting

$$
W^{\frac{\infty}{2}+i}(V)=\oplus_{2 l+j=i} S^{\frac{\infty}{2}+l}(V) \otimes \Lambda^{\frac{\infty}{2}+j}(V) .
$$

Let $V=\mathfrak{g}=\oplus_{n \in \mathbb{Z}} \mathfrak{g}_{n}$ and $\phi$ be the adjoint representation of $\mathfrak{g}$. We define two differentials on the space $W^{\frac{\infty}{2}+*}(\mathfrak{g})$ :

$$
\begin{aligned}
& d=\sum_{i<j}: \tau\left(\left[e_{i}, e_{j}\right]\right) \varepsilon\left(e_{j}^{\prime}\right) \varepsilon\left(e_{i}^{\prime}\right):+\sum_{i, j}: \beta\left(\left[e_{j}, e_{i}\right]\right) \gamma\left(e_{i}^{\prime}\right) \varepsilon\left(e_{j}^{\prime}\right): \\
& \mathbf{h}=\sum_{i} \gamma\left(e_{i}^{\prime}\right) \tau\left(e_{i}\right) .
\end{aligned}
$$

We obtain the semi-infinite Weil complex

$$
\left\{W^{\frac{\infty}{2}+*}(\mathfrak{g}), d+\mathbf{h}\right\}
$$

The differential $d$ is the analogue of the classical differential for the Lie algebra (co)homology, and $h$ is the analogue of the Koszul differential. Notice that

$$
d^{2}=0, \mathrm{~h}^{2}=0,[d, \mathrm{~h}]=0,(d+\mathrm{h})^{2}=0 .
$$

Notice also that if $\mathfrak{g}$ is a finite-dimensional Lie algebra, then applying the definitions given above to the polarization $\mathfrak{g}=\mathfrak{g}_{+} \oplus \mathfrak{g}_{-}$, where $\mathfrak{g}_{+}=\mathfrak{g}, \mathfrak{g}_{-}=0$, we obtain the classical Weil complex.

As in the case of the classical Weil complex, one can construct two filtrations, $F_{1}^{*}$ and $F_{2}^{*}$, on $W^{\frac{\infty}{2}+*}(\mathfrak{g})$ :

$$
F_{1}^{p}=\oplus_{l+j \geq p} S^{\frac{\infty}{2}+l}(\mathfrak{g}) \otimes \Lambda^{\frac{\infty}{2}+j}(\mathfrak{g}), \quad F_{2}^{p}=\oplus_{2 l \geq p} S^{\frac{\infty}{2}+l}(\mathfrak{g}) \otimes \Lambda^{\frac{\infty}{2}+*}(\mathfrak{g}) .
$$

For filtration $F_{1}^{*}$ the complex is acyclic, the second term of the spectral sequence associated to filtration $F_{2}^{*}$ is the semi-infinite cohomology of Lie algebra $\mathfrak{g}$ with coefficients in its "adjoint semi-infinite symmetric powers" $H^{\frac{\infty}{2}+*}\left(\mathfrak{g}, S^{\frac{\infty}{2}+*}(\mathfrak{g})\right)$ (see $\left.[\mathrm{FF}]\right)$. Let

$$
W_{\text {rel }}^{\frac{\infty}{2}+*}(V)=\left\{w \in W^{\frac{\infty}{2}+*}(V) \mid \tau(x) w=0 \text { for all } x \in V_{0}, \theta(x) w=0 \text { for all } x \in \mathfrak{g}_{0}\right\} .
$$

The differential $d$ preserves the space $W_{\text {rel }}^{\frac{\infty}{2}+*}(\mathfrak{g})$ since

$$
[d, \tau(x)]=d \tau(x)+\tau(x) d=\theta(x),
$$


and

$$
[d, \theta(x)]=0
$$

for any $x \in \mathfrak{g}$. The complex $\left\{W_{\text {rel }}^{\frac{\infty}{2}+*}(\mathfrak{g}), d\right\}$ is called the relative semi-infinite Weil complex. Its cohomology is called the relative semi-infinite cohomology $H^{\frac{\infty}{2}+*}\left(\mathfrak{g}, \mathfrak{g}_{0}, S^{\frac{\infty}{2}+*}(\mathfrak{g})\right)$.

We fix $K=0$ from this point on. Correspondingly, $V=V_{+} \oplus V_{-}$, where $V_{+}=\oplus_{n>0} V_{n}$, $V_{-}=\oplus_{n \leq 0} V_{n}$

\section{The $N=2$ superconformal algebra}

Recall that the $N=2 \mathrm{SCA}$ is spanned by the Virasoro generators $\mathcal{L}_{n}$, Heisenberg generators $H_{n}$, two fermionic fields $G_{r}^{ \pm}$, and a central element $\mathrm{C}$, where $n \in \mathbb{Z}, r \in \mathbb{Z}+1 / 2$, and where the non-vanishing commutation relations are as follows, $[\mathrm{FST}]$ :

$$
\begin{aligned}
& {\left[\mathcal{L}_{n}, \mathcal{L}_{m}\right]=(n-m) \mathcal{L}_{n+m}+\frac{\mathrm{C}}{12}\left(n^{3}-n\right) \delta_{n,-m}} \\
& {\left[\mathcal{L}_{n}, H_{m}\right]=-m H_{n+m},\left[\mathcal{L}_{n}, G_{r}^{ \pm}\right]=\left(\frac{n}{2}-r\right) G_{n+r}^{ \pm}} \\
& {\left[G_{r}^{+}, G_{s}^{-}\right]=2 \mathcal{L}_{r+s}+(r-s) H_{r+s}+\frac{\mathrm{C}}{3}\left(r^{2}-\frac{1}{4}\right) \delta_{r,-s}} \\
& {\left[H_{n}, H_{m}\right]=\frac{\mathrm{C}}{3} n \delta_{n,-m},\left[H_{n}, G_{r}^{ \pm}\right]= \pm G_{n+r}^{ \pm}}
\end{aligned}
$$

Let $W i t t=\oplus_{i \in \mathbb{Z}} \mathbb{C} L_{i}$ be the Witt algebra:

$$
\left[L_{i}, L_{j}\right]=(i-j) L_{i+j}
$$

Let $\lambda, \mu \in \mathbb{C}$. Let $\mathcal{F}_{\lambda, \mu}=\oplus_{m \in \mathbb{Z}} \mathbb{C} u_{m}$ be a module over Witt defined as follows:

$$
\phi\left(L_{n}\right) u_{m}=(-m+\mu-(n-1) \lambda) u_{n+m} .
$$

Remark 3.1.- The module $\mathcal{F}_{\lambda, \mu}=\oplus_{m \in \mathbb{Z}} \mathbb{C} u_{m}$ is isomorphic to the module $\mathcal{F}_{-\lambda, \mu+1}=$ $\oplus_{j \in \mathbb{Z}} \mathbb{C} f_{j}$ over the Witt algebra defined in $[\mathrm{Fu}]$. The isomorphism is given by the correspondence $u_{m} \leftrightarrow f_{-m-1}$.

Theorem 3.1.- The space $W^{\frac{\infty}{2}+*}\left(\mathcal{F}_{\lambda, \mu}\right)$ is a module over the $N=2$ SCA with central charge $3-6 \lambda$.

Proof.- Set

$$
\mathrm{h}_{n}=\frac{1}{\sqrt{2}} G_{n-\frac{1}{2}}^{+}, \mathrm{p}_{n}=\frac{1}{\sqrt{2}} G_{n+\frac{1}{2}}^{-} .
$$


We define a representation of Witt in $W^{\frac{\infty}{2}+*}\left(\mathcal{F}_{\lambda, \mu}\right)$ as follows:

$$
\theta\left(L_{n}\right)=\sum_{m \in \mathbb{Z}}(-m+\mu-n \lambda+\lambda)\left(: \tau\left(u_{m+n}\right) \varepsilon\left(u_{m}^{\prime}\right):+: \beta\left(u_{m+n}\right) \gamma\left(u_{m}^{\prime}\right):\right)
$$

Let us extend $\theta$ to a representation of the $N=2 \operatorname{SCA}$ in $W^{\frac{\infty}{2}+*}\left(\mathcal{F}_{\lambda, \mu}\right)$ :

$$
\begin{aligned}
& \theta\left(H_{n}\right)=\lambda \sum_{m \in \mathbb{Z}}: \tau\left(u_{m}\right) \varepsilon\left(u_{m+n}^{\prime}\right):+(\lambda-1) \sum_{m \in \mathbb{Z}}: \beta\left(u_{m}\right) \gamma\left(u_{m+n}^{\prime}\right):+\mu \delta_{n, 0}, \\
& \theta\left(\mathrm{h}_{n}\right)=\sum_{m \in \mathbb{Z}} \gamma\left(u_{m+n}^{\prime}\right) \tau\left(u_{m}\right), \\
& \theta\left(\mathrm{p}_{n}\right)=\sum_{m \in \mathbb{Z}}(m-\mu-(n+1) \lambda) \beta\left(u_{m-n}\right) \varepsilon\left(u_{m}^{\prime}\right), \\
& \theta\left(\mathcal{L}_{n}\right)=-\theta\left(L_{-n}\right)+\frac{n+1}{2} \theta\left(H_{n}\right) .
\end{aligned}
$$

We calculate the central charge by checking the commutation relations on the vacuum vector $\mathbf{\imath}=\mathbf{1}_{S} \otimes \mathbf{1}_{\Lambda}$. Let $n>0$. Then

$$
\begin{aligned}
& \theta\left(\left[H_{n}, H_{-n}\right]\right) \mathbf{1}=-\theta\left(H_{-n}\right) \theta\left(H_{n}\right) \mathbf{1}=-\theta\left(H_{-n}\right)\left(\lambda \sum_{m=1-n}^{0} \tau\left(u_{m}\right) \varepsilon\left(u_{m+n}^{\prime}\right)+\right. \\
& \left.(\lambda-1) \sum_{m=1-n}^{0} \beta\left(u_{m}\right) \gamma\left(u_{m+n}^{\prime}\right)\right) \mathbf{1}=-\lambda^{2} \sum_{m=1-n}^{0} \tau\left(u_{m+n}\right) \varepsilon\left(u_{m}^{\prime}\right) \tau\left(u_{m}\right) \varepsilon\left(u_{m+n}^{\prime}\right) \mathbf{1}- \\
& (\lambda-1)^{2} \sum_{m=1-n}^{0} \beta\left(u_{m+n}\right) \gamma\left(u_{m}^{\prime}\right) \beta\left(u_{m}\right) \gamma\left(u_{m+n}^{\prime}\right) \mathbf{l}=\left(-\lambda^{2} n-(\lambda-1)^{2}(-n)\right) \mathbf{1}=n(1-2 \lambda) \mathbf{1},
\end{aligned}
$$

since $\varepsilon\left(u_{i}^{\prime}\right) \tau\left(u_{i}\right)+\tau\left(u_{i}\right) \varepsilon\left(u_{i}^{\prime}\right)=1$, and $\gamma\left(u_{i}^{\prime}\right) \beta\left(u_{i}\right)-\beta\left(u_{i}\right) \gamma\left(u_{i}^{\prime}\right)=1$. Hence,

$$
\theta\left(\left[H_{n}, H_{m}\right]\right) \mathbf{1}=n(1-2 \lambda) \delta_{n,-m} \mathbf{1} .
$$

Thus the central charge is $3-6 \lambda$. The other commutation relations on the vacuum vector $\mathbf{1}$ are calculated in the same way.

Remark 3.2.- In the case when $\lambda=-1, \mu=1$, the module $\mathcal{F}_{\lambda, \mu}$ is the adjoint representation of Witt. Thus we obtain a representation of the $N=2 \mathrm{SCA}$ in the semi-infinite Weil complex of the Witt algebra (cf. [G]).

Theorem 3.2. Let $V$ be a complex finite-dimensional vector space, $\tilde{V}=V \otimes \mathbb{C}\left[t, t^{-1}\right]$. There exists a representation of the $N=2 \mathrm{SCA}$ in $W^{\frac{\infty}{2}+*}(\tilde{V})$ with central charge $3 \operatorname{dim} V$. 
Proof.- There is the natural $\mathbb{Z}$-grading $\tilde{V}=\oplus_{n \in \mathbb{Z}} \tilde{V}_{n}$, where $\tilde{V}_{n}=V \otimes t^{n}$. Let $u$ run through a fixed basis of $V, u_{n}$ stand for $u \otimes t^{n}$, and let $\left\{u_{n}^{\prime}\right\}$ be the dual basis of $\tilde{V}^{\prime}$. Define the following quadratic expansions by analogy with (3.5) and (3.6), where $\lambda=0, \mu=0$ :

$$
\begin{aligned}
L_{n} & =-\sum_{u} \sum_{m \in \mathbb{Z}}\left(m: \tau\left(u_{m+n}\right) \varepsilon\left(u_{m}^{\prime}\right):+m: \beta\left(u_{m+n}\right) \gamma\left(u_{m}^{\prime}\right):\right) \\
H_{n} & =-\sum_{u} \sum_{m \in \mathbb{Z}}: \gamma\left(u_{m+n}^{\prime}\right) \beta\left(u_{m}\right): \\
\mathrm{h}_{n} & =\sum_{u} \sum_{m \in \mathbb{Z}} \gamma\left(u_{m+n}^{\prime}\right) \tau\left(u_{m}\right), \\
\mathrm{p}_{n} & =\sum_{u} \sum_{m \in \mathbb{Z}} m \beta\left(u_{m-n}\right) \varepsilon\left(u_{m}^{\prime}\right) .
\end{aligned}
$$

Set

$$
\mathcal{L}_{n}=-L_{-n}+\frac{n+1}{2} H_{n}
$$

Then $\mathcal{L}_{n}, H_{n}, \mathrm{~h}_{n}$, and $\mathrm{p}_{n}$ span the centerless $N=2$ SCA.

Let $n>0$. Then $H_{-n} \mathbf{l}=0$. Hence

$$
\begin{aligned}
& {\left[H_{n}, H_{-n}\right] \mathbf{l}=-H_{-n}\left(-\sum_{u} \sum_{m=1-n}^{0} \gamma\left(u_{m+n}^{\prime}\right) \beta\left(u_{m}\right)\right) \mathbf{1}=} \\
& \left(-\sum_{u} \sum_{m=1}^{n} \gamma\left(u_{m-n}^{\prime}\right) \beta\left(u_{m}\right)\right)\left(\sum_{u} \sum_{m=1-n}^{0} \gamma\left(u_{m+n}^{\prime}\right) \beta\left(u_{m}\right)\right) \mathbf{1}= \\
& -\sum_{u} \sum_{m=1-n}^{0} \gamma\left(u_{m}^{\prime}\right) \beta\left(u_{m+n}\right) \gamma\left(u_{m+n}^{\prime}\right) \beta\left(u_{m}\right) \mathbf{1}=-\operatorname{dim} V(-n) \mathbf{1},
\end{aligned}
$$

since $\gamma\left(u_{i}^{\prime}\right) \beta\left(u_{i}\right)-\beta\left(u_{i}\right) \gamma\left(u_{i}^{\prime}\right)=1$. Notice that

$$
\left[H_{n}, H_{m}\right] \mathbf{\imath}=0 \text {, if } m \neq-n
$$

Hence

$$
\left[H_{n}, H_{m}\right] \mathbf{1}=n \operatorname{dim} V \delta_{n,-m} \mathbf{1}
$$

Thus the central charge is $3 \operatorname{dim} V$.

Corollary 3.1.- Let $\mathfrak{g}$ be a complex finite-dimensional Lie algebra, let $\tilde{\mathfrak{g}}=\mathfrak{g} \otimes \mathbb{C}\left[t, t^{-1}\right]$. There exists a representation of the $N=2 \mathrm{SCA}$ in $H^{\frac{\infty}{2}+*}\left(\tilde{\mathfrak{g}}, S^{\frac{\infty}{2}+*}(\tilde{\mathfrak{g}})\right)$ with central charge 3 dimg. 
Proof.- We will show that the expansions (3.9) commute with the differential $d$. Recall that

$$
d=d^{(1)}+d^{(2)}
$$

where

$$
\begin{aligned}
& d^{(1)}=(1 / 2) \sum_{u, v, i, j}: \tau\left(\left[u_{i}, v_{j}\right]\right) \varepsilon\left(v_{j}^{\prime}\right) \varepsilon\left(u_{i}^{\prime}\right): \\
& d^{(2)}=\sum_{u, v, i, j}: \beta\left(\left[u_{i}, v_{j}\right]\right) \gamma\left(v_{j}^{\prime}\right) \varepsilon\left(u_{i}^{\prime}\right):
\end{aligned}
$$

$u, v$ run through a fixed basis of $\mathfrak{g}$, and $i, j \in \mathbb{Z}$. Then

$$
\begin{aligned}
& {\left[L_{n}, d^{(1)}\right]=(1 / 2) \sum_{u, v, i, j}:-(i+j) \tau\left([u, v]_{i+j+n}\right) \varepsilon\left(v_{j}^{\prime}\right) \varepsilon\left(u_{i}^{\prime}\right):+} \\
& : \tau\left(\left[u_{i}, v_{j}\right]\right)(j-n) \varepsilon\left(v_{j-n}^{\prime}\right) \varepsilon\left(u_{i}^{\prime}\right):+: \tau\left(\left[u_{i}, v_{j}\right]\right) \varepsilon\left(v_{j}^{\prime}\right)(i-n) \varepsilon\left(u_{i-n}^{\prime}\right):=0
\end{aligned}
$$

and

$$
\begin{aligned}
& {\left[L_{n}, d^{(2)}\right]=\sum_{u, v, i, j}:-(i+j) \beta\left([u, v]_{i+j+n}\right) \gamma\left(v_{j}^{\prime}\right) \varepsilon\left(u_{i}^{\prime}\right):+} \\
& : \beta\left(\left[u_{i}, v_{j}\right]\right)(j-n) \gamma\left(v_{j-n}^{\prime}\right) \varepsilon\left(u_{i}^{\prime}\right):+: \beta\left(\left[u_{i}, v_{j}\right]\right) \gamma\left(v_{j}^{\prime}\right)(i-n) \varepsilon\left(u_{i-n}^{\prime}\right):=0 .
\end{aligned}
$$

Clearly,

$$
\left[H_{n}, d^{(1)}\right]=0
$$

and

$$
\left[H_{n}, d^{(2)}\right]=\sum_{u, v, i, j}:-\beta\left([u, v]_{i+j-n}\right) \gamma\left(v_{j}^{\prime}\right) \varepsilon\left(u_{i}^{\prime}\right):+\beta\left([u, v]_{i+j}\right) \gamma\left(v_{j+n}^{\prime}\right) \varepsilon\left(u_{i}^{\prime}\right):=0 .
$$

Next,

$$
\begin{aligned}
& {\left[\mathrm{h}_{n}, d^{(1)}\right]=(1 / 2) \sum_{u, v, i, j}:-\tau\left([u, v]_{i+j}\right) \gamma\left(v_{j+n}^{\prime}\right) \varepsilon\left(u_{i}^{\prime}\right):+: \tau\left([u, v]_{i+j}\right) \varepsilon\left(v_{j}^{\prime}\right) \gamma\left(u_{i=n}^{\prime}\right):=} \\
& -\sum_{u, v, i, j}: \tau\left([u, v]_{i+j}\right) \gamma\left(v_{j+n}^{\prime}\right) \varepsilon\left(u_{i}^{\prime}\right): \\
& {\left[\mathrm{h}_{n}, d^{(2)}\right]=\sum_{u, v, i, j}: \tau\left([u, v]_{i+j-n}\right) \gamma\left(v_{j}^{\prime}\right) \varepsilon\left(u_{i}^{\prime}\right):+: \beta\left([u, v]_{i+j}\right) \gamma\left(v_{j}^{\prime}\right) \gamma\left(u_{i+n}^{\prime}\right):=} \\
& \sum_{u, v, i, j}: \tau\left([u, v]_{i+j}\right) \gamma\left(v_{j+n}^{\prime}\right) \varepsilon\left(u_{i}^{\prime}\right):
\end{aligned}
$$


since $\sum_{u, v, i, j}: \beta\left([u, v]_{i+j}\right) \gamma\left(v_{j}^{\prime}\right) \gamma\left(u_{i+n}^{\prime}\right):=0$. Hence

$$
\left[\mathrm{h}_{n}, d^{(2)}\right]=-\left[\mathrm{h}_{n}, d^{(1)}\right]
$$

Finally,

$$
\begin{aligned}
& {\left[\mathrm{p}_{n}, d^{(1)}\right]=(1 / 2) \sum_{u, v, i, j}:(i+j) \beta\left([u, v]_{i+j-n}\right) \varepsilon\left(v_{j}^{\prime}\right) \varepsilon\left(u_{i}^{\prime}\right):} \\
& {\left[\mathrm{p}_{n}, d^{(2)}\right]=\sum_{u, v, i, j}:-\beta\left([u, v]_{i+j}\right)(j+n) \varepsilon\left(v_{j+n}^{\prime}\right) \varepsilon\left(u_{i}^{\prime}\right):=} \\
& \sum_{u, v, i, j}:-\beta\left([u, v]_{i+j-n}\right) j \varepsilon\left(v_{j}^{\prime}\right) \varepsilon\left(u_{i}^{\prime}\right):=-(1 / 2) \sum_{u, v, i, j}:(j+i) \beta\left([u, v]_{i+j-n}\right) \varepsilon\left(v_{j}^{\prime}\right) \varepsilon\left(u_{i}^{\prime}\right): .
\end{aligned}
$$

Hence

$$
\left[\mathrm{p}_{n}, d^{(2)}\right]=-\left[\mathrm{p}_{n}, d^{(1)}\right]
$$

\section{The superconformal algebras $S^{\prime}(2, \alpha)$}

Recall the necessary definitions, $[\mathrm{KL}]$. Let $W(N)$ be the superalgebra of all derivations of $\mathbb{C}\left[t, t^{-1}\right] \otimes \Lambda(N)$, where $\Lambda(N)$ is the Grassmann algebra in $N$ variables $\theta_{1}, \ldots, \theta_{N}$, and $p(t)=\overline{0}, p\left(\theta_{i}\right)=\overline{1}$ for $i=1, \ldots, N$. Let $\partial_{i}$ stand for $\partial / \partial \theta_{i}$, and $\partial_{t}$ stand for $\partial / \partial t$. Let

$$
S(N, \alpha)=\left\{D \in W(N) \mid \operatorname{Div}\left(t^{\alpha} D\right)=0\right\} \text { for } \alpha \in \mathbb{C}
$$

Recall that

$$
\operatorname{Div}\left(f \partial_{t}+\sum_{i=1}^{N} f_{i} \partial_{i}\right)=\partial_{t} f+\sum_{i=1}^{N}(-1)^{p\left(f_{i}\right)} \partial_{i} f_{i}
$$

where $f, f_{i} \in \mathbb{C}\left[t, t^{-1}\right] \otimes \Lambda(N)$, and

$$
\operatorname{Div}(f D)=D f+f D i v D
$$

where $f$ is an even function. Let $S^{\prime}(N, \alpha)=[S(N, \alpha), S(N, \alpha)]$ be the derived superalgebra. Assume that $N>1$. If $\alpha \notin \mathbb{Z}$, then $S(N, \alpha)$ is simple, and if $\alpha \in \mathbb{Z}$, then $S^{\prime}(N, \alpha)$ is a simple ideal of $S(N, \alpha)$ of codimension 1 :

$$
0 \rightarrow S^{\prime}(N, \alpha) \rightarrow S(N, \alpha) \rightarrow \mathbb{C} t^{-\alpha} \theta_{1} \cdots \theta_{N} \partial_{t} \rightarrow 0
$$


Notice that

$$
S(N, \alpha) \cong S(N, \alpha+n) \text { for } n \in \mathbb{Z} .
$$

The superalgebra $S^{\prime}(N, \alpha)$ has, up to equivalence, only one non-trivial cocycle if and only if $N=2$, which is important for our task. Let

$$
\left\{\mathcal{L}_{n}^{\alpha}, E_{n}, H_{n}, F_{n}, \mathrm{~h}_{n}^{\alpha}, \mathrm{p}_{n}, \mathrm{x}_{n}, \mathrm{y}_{n}^{\alpha}\right\}_{n \in \mathbb{Z}}
$$

be the basis of $S^{\prime}(2, \alpha)$ defined as follows:

$$
\begin{aligned}
& \mathcal{L}_{n}^{\alpha}=-t^{n}\left(t \partial_{t}+\frac{1}{2}(n+\alpha+1)\left(\theta_{1} \partial_{1}+\theta_{2} \partial_{2}\right)\right) \\
& E_{n}=t^{n} \theta_{2} \partial_{1} \\
& H_{n}=t^{n}\left(\theta_{2} \partial_{2}-\theta_{1} \partial_{1}\right) \\
& F_{n}=t^{n} \theta_{1} \partial_{2} \\
& \mathrm{~h}_{n}^{\alpha}=t^{n} \theta_{2} \partial_{t}-(n+\alpha) t^{n-1} \theta_{1} \theta_{2} \partial_{1} \\
& \mathrm{p}_{n}=-t^{n+1} \partial_{2} \\
& x_{n}=t^{n+1} \partial_{1} \\
& \mathrm{y}_{n}^{\alpha}=t^{n} \theta_{1} \partial_{t}+(n+\alpha) t^{n-1} \theta_{1} \theta_{2} \partial_{2}
\end{aligned}
$$

The non-vanishing commutation relations between these elements are

$$
\begin{aligned}
& {\left[\mathcal{L}_{n}^{\alpha}, \mathcal{L}_{k}^{\alpha}\right]=(n-k) \mathcal{L}_{n+k}^{\alpha},} \\
& {\left[E_{n}, F_{k}\right]=H_{n+k},\left[H_{n}, E_{k}\right]=2 E_{n+k},\left[H_{n}, F_{k}\right]=-2 F_{n+k} \text {, }} \\
& {\left[\mathcal{L}_{n}^{\alpha}, E_{k}\right]=-k E_{n+k},\left[\mathcal{L}_{n}^{\alpha}, H_{k}\right]=-k H_{n+k},\left[\mathcal{L}_{n}^{\alpha}, F_{k}\right]=-k F_{n+k},} \\
& {\left[\mathcal{L}_{n}^{\alpha}, \mathrm{h}_{k}^{\alpha}\right]=\frac{1}{2}(n-2 k+1-\alpha) \mathrm{h}_{n+k}^{\alpha},\left[\mathcal{L}_{n}^{\alpha}, \mathrm{p}_{k}\right]=\frac{1}{2}(n-2 k-1+\alpha) \mathrm{p}_{n+k},} \\
& {\left[\mathcal{L}_{n}^{\alpha}, x_{k}\right]=\frac{1}{2}(n-2 k-1+\alpha) x_{n+k},\left[\mathcal{L}_{n}^{\alpha}, y_{k}^{\alpha}\right]=\frac{1}{2}(n-2 k+1-\alpha) y_{n+k}^{\alpha},} \\
& {\left[E_{n}, \mathrm{y}_{k}^{\alpha}\right]=\mathrm{h}_{n+k}^{\alpha},\left[F_{n}, \mathrm{~h}_{k}^{\alpha}\right]=\mathrm{y}_{n+k}^{\alpha},\left[E_{n}, \mathrm{p}_{k}\right]=\mathrm{x}_{n+k},\left[F_{n}, \mathrm{x}_{k}\right]=\mathrm{p}_{n+k},} \\
& {\left[H_{n}, \mathrm{~h}_{k}^{\alpha}\right]=\mathrm{h}_{n+k}^{\alpha},\left[H_{n}, \mathrm{y}_{k}^{\alpha}\right]=-\mathrm{y}_{n+k}^{\alpha},\left[H_{n}, \mathrm{x}_{k}\right]=\mathrm{x}_{n+k},\left[H_{n}, \mathrm{p}_{k}\right]=-\mathrm{p}_{n+k},} \\
& {\left[\mathrm{~h}_{n}^{\alpha}, \mathrm{x}_{k}\right]=(k+1-n-\alpha) E_{n+k},\left[\mathrm{p}_{n}, \mathrm{y}_{k}^{\alpha}\right]=(k-n-1+\alpha) F_{n+k},} \\
& {\left[\mathrm{~h}_{n}^{\alpha}, \mathrm{p}_{k}\right]=\mathcal{L}_{n+k}^{\alpha}-\frac{1}{2}(k-n+1-\alpha) H_{n+k},} \\
& {\left[\mathrm{x}_{n}, \mathrm{y}_{k}^{\alpha}\right]=-\mathcal{L}_{n+k}^{\alpha}+\frac{1}{2}(k-n-1+\alpha) H_{n+k} \text {. }}
\end{aligned}
$$


A non-trivial cocycle on $S^{\prime}(2, \alpha)$ is

$$
\begin{aligned}
& c\left(\mathcal{L}_{n}^{\alpha}, \mathcal{L}_{k}^{\alpha}\right)=\frac{\mathrm{C}}{12} n\left(n^{2}-1\right) \delta_{n,-k}, \\
& c\left(E_{n}, F_{k}\right)=\frac{\mathrm{C}}{6} n \delta_{n,-k}, c\left(H_{n}, H_{k}\right)=\frac{\mathrm{C}}{3} n \delta_{n,-k}, \\
& c\left(\mathrm{~h}_{n}^{\alpha}, \mathrm{p}_{k}\right)=\frac{\mathrm{C}}{6}\left(\left(n-1+\frac{\alpha+1}{2}\right)^{2}-\frac{1}{4}\right) \delta_{n,-k}, \\
& c\left(\mathrm{x}_{n}, \mathrm{y}_{k}^{\alpha}\right)=-\frac{\mathrm{C}}{6}\left(\left(-n-1+\frac{\alpha+1}{2}\right)^{2}-\frac{1}{4}\right) \delta_{n,-k} ;
\end{aligned}
$$

see $[\mathrm{KL}]$. Let $\hat{S}^{\prime}(2, \alpha)$ be the corresponding central extension of $S^{\prime}(2, \alpha)$. In particular, $\hat{S}^{\prime}(2,0)$ is isomorphic to the $N=4$ SCA (see [Ad]).

Remark 4.1.- Notice that

$$
\begin{aligned}
& S^{\prime}(2, \alpha)_{\overline{0}}=W i t t \ltimes \tilde{\mathfrak{s l}}(2), \text { where } \\
& W i t t=\left\langle\mathcal{L}_{n}^{\alpha}\right\rangle_{n \in \mathbb{Z}}, \tilde{\mathfrak{s l}}(2)=\left\langle E_{n}, H_{n}, F_{n}\right\rangle_{n \in \mathbb{Z}},
\end{aligned}
$$

and

$$
S^{\prime}(2, \alpha)_{\overline{1}}=\left\langle\mathrm{h}_{n}^{\alpha}, \mathrm{y}_{n}^{\alpha}\right\rangle_{n \in \mathbb{Z}} \oplus\left\langle\mathrm{p}_{n}, x_{n}\right\rangle_{n \in \mathbb{Z}}
$$

is a direct sum of two standard (odd) $\tilde{\mathfrak{s}} \mathfrak{l}(2)$-modules.

Remark 4.2.- For any $\alpha \in \mathbb{C}$ one can consider the subalgebra of $\hat{S}^{\prime}(2, \alpha)$, spanned by $\mathcal{L}_{n}^{\alpha}, H_{n}, \mathrm{~h}_{n}^{\alpha}, \mathrm{p}_{n}$, and C. Thus we obtain a one-parameter family of superalgebras, which are isomorphic to the $N=2 \mathrm{SCA}$. The isomorphism

$$
\varphi:\left\langle\mathcal{L}_{n}^{\alpha}, H_{n}, \mathrm{~h}_{n}^{\alpha}, \mathrm{p}_{n}, \mathrm{C}\right\rangle \longrightarrow\left\langle\mathcal{L}_{n}, H_{n}, \mathrm{~h}_{n}, \mathrm{p}_{n}, \mathrm{C}\right\rangle
$$

is given as follows:

$$
\begin{aligned}
& \varphi\left(\mathcal{L}_{n}^{\alpha}\right)=\mathcal{L}_{n}-\frac{\alpha}{2} H_{n}+\frac{\alpha^{2}}{24} \delta_{n, 0} \mathrm{C}, \\
& \varphi\left(H_{n}\right)=H_{n}-\frac{\alpha}{6} \delta_{n, 0} \mathrm{C}, \\
& \varphi\left(\mathrm{h}_{n}^{\alpha}\right)=\mathrm{h}_{n}, \varphi\left(\mathrm{p}_{n}\right)=\mathrm{p}_{n}, \varphi(\mathrm{C})=\mathrm{C} .
\end{aligned}
$$

Notice that formulae (4.13) correspond to the spectral flow transformation for the $N=2$ SCA (cf. [FST]).

Let $\operatorname{Der} S^{\prime}(2, \alpha)$ be the Lie superalgebra of all derivations of $S^{\prime}(2, \alpha)$, and $D e r_{e x t} S^{\prime}(2, \alpha)$ be the exterior derivations of $S^{\prime}(2, \alpha)$ (see $[\mathrm{Fu}]$ ). 
Theorem 4.1.-

1) If $\alpha \in \mathbb{Z}$, then $\operatorname{Der}_{\text {ext }} S^{\prime}(2, \alpha) \cong \mathcal{S} \mathcal{L}(2)=\langle\mathcal{E}, \mathcal{H}, \mathcal{F}\rangle$, where

$$
[\mathcal{H}, \mathcal{E}]=2 \mathcal{E},[\mathcal{H}, \mathcal{F}]=-2 \mathcal{F},[\mathcal{E}, \mathcal{F}]=\mathcal{H}
$$

The action of $\mathcal{S} \mathcal{L}(2)$ is given as follows:

$$
\begin{aligned}
& {\left[\mathcal{E}, \mathrm{h}_{k}^{\alpha}\right]=\mathrm{x}_{k-1+\alpha},\left[\mathcal{E}, \mathrm{y}_{k}^{\alpha}\right]=\mathrm{p}_{k-1+\alpha}} \\
& {\left[\mathcal{F}, \boldsymbol{x}_{k}\right]=\mathrm{h}_{k+1-\alpha}^{\alpha},\left[\mathcal{F}, \mathrm{p}_{k}\right]=\mathrm{y}_{k+1-\alpha}^{\alpha} ;} \\
& {\left[\mathcal{H}, \boldsymbol{x}_{k}\right]=\mathrm{x}_{k},\left[\mathcal{H}, \mathrm{h}_{k}^{\alpha}\right]=-\mathrm{h}_{k}^{\alpha}} \\
& {\left[\mathcal{H}, \mathrm{p}_{k}\right]=\mathrm{p}_{k},\left[\mathcal{H}, \mathrm{y}_{k}^{\alpha}\right]=-\mathrm{y}_{k}^{\alpha} .}
\end{aligned}
$$

2) If $\alpha \in \mathbb{C} \backslash \mathbb{Z}$, then $\operatorname{Der}_{\text {ext }} S^{\prime}(2, \alpha)=\langle\mathcal{H}\rangle$.

Proof.- Recall that the exterior derivations of a Lie (super) algebra can be identified with its first cohomology with coefficients in the adjoint representation (see [Fu]). Thus

$$
\operatorname{Der}_{e x t} S^{\prime}(2, \alpha) \cong H^{1}\left(S^{\prime}(2, \alpha), S^{\prime}(2, \alpha)\right)
$$

The superalgebra $S^{\prime}(2, \alpha)$ has the following $\mathbb{Z} \pm \alpha$-grading deg:

$$
\begin{aligned}
& \operatorname{deg} \mathcal{L}_{n}^{\alpha}=n, \operatorname{deg} E_{n}=n+1-\alpha, \operatorname{deg} F_{n}=n-1+\alpha, \operatorname{deg} H_{n}=n \\
& \operatorname{deg} \mathrm{h}_{n}^{\alpha}=n, \operatorname{deg} \mathrm{p}_{n}=n, \operatorname{deg} x_{n}=n+1-\alpha, \operatorname{deg} \mathbf{y}_{n}^{\alpha}=n-1+\alpha .
\end{aligned}
$$

Let

$$
L_{0}=-\mathcal{L}_{0}^{\alpha}+\frac{1}{2}(1-\alpha) H_{0}
$$

Then

$$
\left[L_{0}, s\right]=(\text { degs }) s
$$

for a homogeneous $s \in S^{\prime}(2, \alpha)$. Accordingly,

$$
\left[L_{0}, D\right]=(\operatorname{deg} D) D
$$

for a homogeneous $D \in \operatorname{Der}_{\text {ext }} S^{\prime}(2, \alpha)$. On the other hand, since the action of a Lie superalgebra on its cohomology is trivial (see $[\mathrm{Fu}]$ ), then one must have

$$
\left[L_{0}, D\right]=0
$$


Hence the non-zero elements of $\operatorname{Der}_{e x t} S^{\prime}(2, \alpha)$ have $d e g=0$, and they preserve the superalgebra $S^{\prime}(2, \alpha)_{\operatorname{deg}=0}$. Let $\alpha \in \mathbb{Z}$. Then one can check that the exterior derivations of $S^{\prime}(2, \alpha)_{\operatorname{deg}=0}$ form an $\mathfrak{s l}(2)$, and extend them to the exterior derivations of $S^{\prime}(2, \alpha)$ as in (4.15). One should also note that if the restriction of a derivation of $S^{\prime}(2, \alpha)$ to $S^{\prime}(2, \alpha)_{\operatorname{deg}=0}$ is zero, then this derivation is inner.

Finally, notice that the exterior derivations $\mathcal{E}$ and $\mathcal{F}$ interchange $\left\{\mathrm{h}_{k}^{\alpha}\right\}$ with $\left\{x_{k}\right\}$. If $\alpha \notin \mathbb{Z}$, then $\operatorname{deg} \mathrm{h}_{k}^{\alpha}-\operatorname{deg} \boldsymbol{x}_{n} \notin \mathbb{Z}$ for any $k, n \in \mathbb{Z}$. Hence $\mathcal{E}$ and $\mathcal{F}$ cannot have deg $=0$. By this reason, $\operatorname{Der}_{\text {ext }} S^{\prime}(2, \alpha)=\langle\mathcal{H}\rangle$ for $\alpha \in \mathbb{C} \backslash \mathbb{Z}$.

Remark 4.3.- If $\alpha \in \mathbb{Z}$, then one can identify $\mathcal{F}$ with $-t^{-\alpha} \theta_{1} \theta_{2} \partial_{t}$ (see (4.4)).

\section{An action of $\hat{S}^{\prime}(2, \alpha)$ on the semi-infinite Weil complex of a loop algebra}

We will consider a more general case, i. e. when $V$ is a complex finite-dimensional vector space, and $\tilde{V}=V \otimes \mathbb{C}\left[t, t^{-1}\right]$. Let $\hat{D} e r S^{\prime}(2, \alpha)$ be a non-trivial central extension of $\operatorname{Der} S^{\prime}(2, \alpha)$.

Theorem 5.1.-

1) The space $W^{\frac{\infty}{2}+*}(\tilde{V})$, where $\alpha \in \mathbb{C}$, is a module over $\hat{S}^{\prime}(2, \alpha)$ with central charge $3 \operatorname{dim} V$;

2) if $\alpha \in \mathbb{C} \backslash \mathbb{Z}$, then $W^{\frac{\infty}{2}+*}(\tilde{V})$ is a module over $\hat{\operatorname{Der}} S^{\prime}(2, \alpha)$.

Proof. Let $u$ run through a fixed basis of $V, u_{n}$ stand for $u \otimes t^{n}$, and $\left\{u_{n}^{\prime}\right\}$ be the dual basis of $\tilde{V}^{\prime}$. One can define a representation of Witt in $W^{\frac{\infty}{2}+*}(\tilde{V})$ by analogy with (3.5), where $\lambda=0, \mu=\alpha / 2$ :

$$
\theta\left(L_{n}\right)=-\sum_{u} \sum_{m}\left(m-\frac{\alpha}{2}\right)\left(: \tau\left(u_{m+n}\right) \varepsilon\left(u_{m}^{\prime}\right):+: \beta\left(u_{m+n}\right) \gamma\left(u_{m}^{\prime}\right):\right),
$$

then extend it to a representation of the $N=2 \mathrm{SCA}$, and apply (4.13). We obtain the following representation of $\hat{S}^{\prime}(2, \alpha)$ :

$$
\begin{aligned}
& \theta\left(H_{n}\right)=-\sum_{u} \sum_{m}: \beta\left(u_{m}\right) \gamma\left(u_{m+n}^{\prime}\right), \\
& \theta\left(\mathcal{L}_{n}^{\alpha}\right)=-\theta\left(L_{-n}\right)+\frac{n+1-\alpha}{2} \theta\left(H_{n}\right)+\left(\frac{\alpha}{4}-\frac{\alpha^{2}}{8}\right) \operatorname{dim} V \delta_{n, 0}, \\
& \theta\left(\mathrm{h}_{n}^{\alpha}\right)=\sum_{u} \sum_{m} \gamma\left(u_{m+n}^{\prime}\right) \tau\left(u_{m}\right),
\end{aligned}
$$




$$
\begin{aligned}
& \theta\left(\mathrm{p}_{n}\right)=\sum_{u} \sum_{m}\left(m-\frac{\alpha}{2}\right) \beta\left(u_{m-n}\right) \varepsilon\left(u_{m}^{\prime}\right), \\
& \theta\left(E_{n}\right)=-(1 / 2) i \sum_{u} \sum_{m} \gamma\left(u_{m}^{\prime}\right) \gamma\left(u_{1-m+n}^{\prime}\right), \\
& \theta\left(F_{n}\right)=-(1 / 2) i \sum_{u} \sum_{m} \beta\left(u_{m}\right) \beta\left(u_{1-m-n}\right), \\
& \theta\left(\mathbf{y}_{n}^{\alpha}\right)=i \sum_{u} \sum_{m} \beta\left(u_{m}\right) \tau\left(u_{1-m-n}\right), \\
& \theta\left(x_{n}\right)=-i \sum_{u} \sum_{m}\left(m-\frac{\alpha}{2}\right) \gamma\left(u_{1-m+n}^{\prime}\right) \varepsilon\left(u_{m}^{\prime}\right), \\
& \theta(\mathcal{H})=-\sum_{u} \sum_{m}: \tau\left(u_{m}\right) \varepsilon\left(u_{m}^{\prime}\right) .
\end{aligned}
$$

One can check that the central charge is $3 \operatorname{dim} V$ in the same way as in Theorem 3.2 .

Theorem 5.2.- Let $\mathfrak{g}$ be a complex finite-dimensional Lie algebra endowed with a nondegenerate invariant symmetric bilinear form. Then $H^{\frac{\infty}{2}+*}\left(\tilde{\mathfrak{g}}, S^{\frac{\infty}{2}+*}(\tilde{\mathfrak{g}})\right)$ is a module over $\hat{S}^{\prime}(2,0)$ with central charge 3 dim $\mathfrak{g}$.

Proof.- Let $\left\{v_{i}\right\}$ be a basis of $\mathfrak{g}$ so that with respect to the given form $\left\langle v_{i}, v_{j}\right\rangle=\delta_{i, j}$. Let $u$ run through this basis. Then by Theorem 5.1, there is a representation of $\hat{S}^{\prime}(2,0)$ in $W^{\frac{\infty}{2}+*}(\tilde{\mathfrak{g}})$. Notice that we can identify the elements of $S^{\prime}(2,0)$ with the quadratic expansions obtained by putting $\alpha=0$ in the equations (5.2). One can check that the commutation relations (4.8) (where $\alpha=0$ ) are fulfilled. One can notice that

$$
\left[S^{\prime}(2,0), d\right]=0
$$

In fact, since $\langle\cdot, \cdot\rangle$ is an invariant symmetric bilinear form on $\mathfrak{g}$, then the elements $E_{n}, H_{n}$, and $F_{n}$ commute with $\pi(g)$ for any $g \in \tilde{\mathfrak{g}}$. Hence they commute with $d$. According to Corollary 3.1,

$$
\left[\mathrm{h}_{n}^{0}, d\right]=\left[\mathrm{p}_{n}, d\right]=0
$$

Recall that

$$
S^{\prime}(2,0)_{\overline{1}}=\left\langle h_{n}^{0}, y_{n}^{0}, p_{n}, x_{n}\right\rangle_{n \in \mathbb{Z}}
$$

Since

$$
\left[E_{n}, \mathrm{p}_{k}\right]=x_{n+k},\left[F_{n}, \mathrm{~h}_{k}^{0}\right]=\mathrm{y}_{n+k}^{0}
$$

then

$$
\left[S^{\prime}(2,0)_{\overline{1}}, d\right]=0 \text {. }
$$


Since

$$
S^{\prime}(2,0)_{\overline{0}}=\left[S^{\prime}(2,0)_{\overline{1}}, S^{\prime}(2,0)_{\overline{1}}\right],
$$

then (5.3) follows.

To define an action of $\hat{D} e r S^{\prime}(2,0)$, one should consider a relative semi-infinite Weil complex.

Let $\mathfrak{g}$ be a complex finite-dimensional Lie algebra, $\phi$ be a representation of $\mathfrak{g}$ in $V$, $\langle\cdot, \cdot\rangle$ be a non-degenerate $\mathfrak{g}$-invariant symmetric bilinear form on $V$. One can naturally extend $\phi$ to a representation of $\tilde{\mathfrak{g}}$ in $\tilde{V}$ :

$$
\phi\left(g \otimes t^{n}\right)\left(v \otimes t^{k}\right)=(\phi(g) v) \otimes t^{n+k}, \text { for } g \in \mathfrak{g}, v \in V .
$$

Theorem 5.3.- The space $W_{r e l}^{\frac{\infty}{2}+*}(\tilde{V})$ is a module over $\hat{D} e r S^{\prime}(2,0)$ with central charge $3 \operatorname{dim} V$.

Proof.- Let $\left\{v_{i}\right\}$ be a basis of $V$ so that $\left\langle v_{i}, v_{j}\right\rangle=\delta_{i, j}$. Let $u$ run through this basis. Then by Theorem 5.1, there is a representation of $\hat{S}^{\prime}(2,0)$ in $W^{\frac{\infty}{2}+*}(\tilde{V})$. We can identify the elements of $S^{\prime}(2,0)$ with the expansions (5.2) where $\alpha=0$.

Since the form $\langle\cdot, \cdot\rangle$ is $\mathfrak{g}$-invariant, then there is an action of $\hat{S}^{\prime}(2,0)$ on $W_{\text {rel }}^{\frac{\infty}{2}+*}(\tilde{V})$. To extend this representation to $\hat{D} e r S^{\prime}(2,0)$, we have to define it on $\mathcal{S} \mathcal{L}(2)=\langle\mathcal{F}, \mathcal{H}, \mathcal{E}\rangle$. Let

$$
\begin{aligned}
\mathcal{E} & =i \sum_{u} \sum_{m>0} m \varepsilon\left(u_{-m}^{\prime}\right) \varepsilon\left(u_{m}^{\prime}\right), \\
\mathcal{H} & =-\sum_{u} \sum_{m \neq 0}: \tau\left(u_{m}\right) \varepsilon\left(u_{m}^{\prime}\right):, \\
\mathcal{F} & =-i \sum_{u} \sum_{m>0}(1 / m) \tau\left(u_{m}\right) \tau\left(u_{-m}\right) .
\end{aligned}
$$

Notice that $\mathcal{S} \mathcal{L}(2)$ acts on $W_{\text {rel }}^{\frac{\infty}{2}+*}(\tilde{V})$. The commutation relations between $\mathcal{E}, \mathcal{H}, \mathcal{F}$ and the elements of $S^{\prime}(2,0)$ coincide with the relations (4.15), where $\alpha=0$, up to some terms which contain elements $\tau\left(u_{0}\right)$. Since the action of $\tau\left(u_{0}\right)$ on $W_{r e l}^{\frac{\infty}{2}+*}(\tilde{V})$ is trivial, then a representation of $\hat{D} e r S^{\prime}(2,0)$ in $W_{\text {rel }}^{\frac{\infty}{2}+*}(\tilde{V})$ is well-defined.

Corollary 5.1.- $H^{\frac{\infty}{2}+*}\left(\tilde{\mathfrak{g}}, \tilde{\mathfrak{g}}_{0}, S^{\frac{\infty}{2}+*}(\tilde{\mathfrak{g}})\right)$ is a module over $\hat{S}^{\prime}(2,0)$ with central charge $3 \operatorname{dim} \mathfrak{g}$. 
Proof.- Follows from Theorem 5.2.

\section{Relative semi-infinite cohomology and Kähler geometry}

Let $M$ be a compact Kähler manifold with associated $(1,1)$-form $\omega$, let $\operatorname{dim}_{\mathbb{C}} M=n$. There exists a number of operators on the space $A^{*}(M)$ of differential forms on $M$ such as $\partial, \bar{\partial}, d, d_{c}$, their corresponding adjoint operators and the associated Laplacians (see [GH]). Recall that

$$
\begin{aligned}
& \partial: A^{p, q}(M) \rightarrow A^{p+1, q}(M), \\
& \bar{\partial}: A^{p, q}(M) \rightarrow A^{p, q+1}(M), \\
& d=\partial+\bar{\partial} \\
& d_{c}=i(\partial-\bar{\partial}), \\
& \triangle=d d^{*}+d^{*} d=2 \triangle_{\partial}=2 \triangle_{\bar{\partial}} .
\end{aligned}
$$

The Hodge $\star$-operator maps

$$
\star: A^{p, q}(M) \longrightarrow A^{n-q, n-p}(M),
$$

so that $\star^{2}=(-1)^{p+q}$ on $A^{p, q}(M)$. Correspondingly, the Hodge inner product is defined on each of $A^{p, q}(M)$ :

$$
(\varphi, \psi)=\int_{M} \varphi \wedge \star \bar{\psi} .
$$

In addition, $A^{*}(M)$ admits an $\mathfrak{s l}(2)$-module structure. Namely, $\mathfrak{s l}(2)=\langle L, H, \Lambda\rangle$, where

$$
[L, \Lambda]=H,[H, L]=2 L,[H, \Lambda]=-2 \Lambda .
$$

The operator

$$
L: A^{p, q}(M) \rightarrow A^{p+1, q+1}(M),
$$

is defined by

$$
L(\varphi)=\varphi \wedge \omega .
$$

Let $\Lambda=L^{*}$ be its adjoint operator:

$$
\Lambda: A^{p, q}(M) \rightarrow A^{p-1, q-1}(M),
$$


and

$$
\left.H\right|_{A^{p, q}(M)}=p+q-n
$$

According to the Lefschetz theorem, there exists the corresponding action of $\mathfrak{s l}(2)$ on $H^{*}(M)$. These operators satisfy a series of identities, known as the Hodge identities (see $[\mathrm{GH}])$. Consider the Lie superalgebra spanned by the classical operators:

$$
\mathcal{S}:=\left\langle\triangle, L, H, \Lambda, d, d^{*}, d_{c}, d_{c}^{*}\right\rangle
$$

The non-vanishing commutation relations in $\mathcal{S}$ are as follows:

$$
\begin{aligned}
& {[L, \Lambda]=H,[H, L]=2 L,[H, \Lambda]=-2 \Lambda,} \\
& {\left[d, d^{*}\right]=d d^{*}+d^{*} d=\triangle,} \\
& {\left[d_{c}, d_{c}^{*}\right]=d_{c} d_{c}^{*}+d_{c}^{*} d_{c}=\triangle,} \\
& {[H, d]=d,\left[H, d^{*}\right]=-d^{*},} \\
& {\left[H, d_{c}\right]=d_{c},\left[H, d_{c}^{*}\right]=-d_{c}^{*},} \\
& {\left[L, d^{*}\right]=-d_{c},\left[L, d_{c}^{*}\right]=d,} \\
& {[\Lambda, d]=d_{c}^{*},\left[\Lambda, d_{c}\right]=-d^{*} .}
\end{aligned}
$$

Theorem 6.1.- Let $\mathfrak{g}$ be a complex finite-dimensional Lie algebra with a non-degenerate invariant symmetric bilinear form. Then there exist operators on $W_{r e l}^{\frac{\infty}{2}+*}(\tilde{\mathfrak{g}})$, which are analogous to the classical operators in Kähler geometry.

Proof.- It was shown in [FGZ] that a relative semi-infinite complex $C_{\infty}^{*}\left(\mathfrak{l}, \mathfrak{l}_{0}, V\right)$, where $\mathfrak{l}=\oplus_{n \in \mathbb{Z}^{\mathfrak{l}}}$ is a complex $\mathbb{Z}$-graded Lie algebra, and $V$ is a graded Hermitian $\mathfrak{l}$-module, has a structure, which is similar to that of the de Rham complex in Kähler geometry. It is assumed that there exists a 2-cocycle $\gamma$ on $\mathfrak{l}$ such that $\left.\gamma\right|_{\mathfrak{l}_{n} \times \mathfrak{l}_{-n}}$ is non-degenerate if $n \in \mathbb{Z} \backslash 0$ and it is zero otherwise. Then there exist operators on $C_{\infty}^{*}\left(\mathfrak{l}, \mathfrak{l}_{0}, V\right)$ analogous to the classical ones.

We will define analogues of the classical operators on $W_{r e l}^{\frac{\infty}{2}+*}(\tilde{\mathfrak{g}})$. Using the form $\langle\cdot, \cdot\rangle$ on $\mathfrak{g}$ we obtain the 2 -cocycle $\gamma$ on $\tilde{\mathfrak{g}}$ :

$$
\gamma\left(g_{1} \otimes t^{n}, g_{2} \otimes t^{m}\right)=n\left\langle g_{1}, g_{2}\right\rangle \delta_{n,-m}, \text { for } g_{1}, g_{2} \in \mathfrak{g}
$$

Notice that $\gamma \mid \tilde{\mathfrak{g}}_{n} \times \tilde{\mathfrak{g}}_{-n}$ is non-degenerate if $n \in \mathbb{Z} \backslash 0$ and zero otherwise. Let

$$
\Lambda_{r e l}^{\frac{\infty}{2}+*}(\tilde{\mathfrak{g}})=\oplus_{a, b \geq 0} \Lambda^{a}\left(\mathfrak{n}_{+}^{\prime}\right) \wedge \Lambda_{\infty}^{b}\left(\mathfrak{n}_{-}^{\prime}\right)
$$


For a homogeneous element in $\Lambda^{a}\left(\mathfrak{n}_{+}^{\prime}\right) \wedge \Lambda_{\infty}^{b}\left(\mathfrak{n}_{-}^{\prime}\right), a$ is the number of added elements, and $b$ is the number of missing elements with respect to the vacuum vector $\mathbf{1}_{r e l}$. Let

$$
C^{a, b}(\tilde{\mathfrak{g}})=\left[S^{\frac{\infty}{2}+*}(\tilde{\mathfrak{g}}) \otimes \Lambda^{a}\left(\mathfrak{n}_{+}^{\prime}\right) \wedge \Lambda_{\infty}^{b}\left(\mathfrak{n}_{-}^{\prime}\right)\right]^{\mathfrak{g}_{0}}
$$

We obtain a bigrading on the relative semi-infinite Weil complex, such that

$$
W_{r e l}^{\frac{\infty}{2}+i}(\tilde{\mathfrak{g}})=\oplus_{a-b=i} C^{a, b}(\tilde{\mathfrak{g}})
$$

Let $d$ be the restriction of the differential to the relative subcomplex. Notice that

$$
d: C^{a, b}(\tilde{\mathfrak{g}}) \longrightarrow C^{a+1, b}(\tilde{\mathfrak{g}}) \oplus C^{a, b-1}(\tilde{\mathfrak{g}}) .
$$

Define $d_{1}$ and $d_{2}$ such that

$$
\begin{aligned}
& d=d_{1}+d_{2}, \\
& d_{1}: C^{a, b}(\tilde{\mathfrak{g}}) \longrightarrow C^{a+1, b}(\tilde{\mathfrak{g}}), \\
& d_{2}: C^{a, b}(\tilde{\mathfrak{g}}) \longrightarrow C^{a, b-1}(\tilde{\mathfrak{g}}) .
\end{aligned}
$$

Let

$$
d_{c}=i\left(d_{1}-d_{2}\right)
$$

To define the adjoint operators, we have to introduce a Hermitian form on $W_{r e l}^{\frac{\infty}{2}+*}(\tilde{\mathfrak{g}})$.

It was shown in [FGZ] that if a $\mathbb{Z}$-graded Lie algebra $\mathfrak{l}$ admits an antilinear automorphism $\sigma$ of order 2 such that $\sigma\left(\mathfrak{l}_{n}\right)=\mathfrak{l}_{-n}$, then there exists a Hermitian form on $\Lambda^{\frac{\infty}{2}+*}(\mathfrak{l})$ such that

$$
\varepsilon\left(x^{\prime}\right)^{*}=-\varepsilon\left(\sigma\left(x^{\prime}\right)\right), \quad \tau(x)^{*}=-\tau(\sigma(x)),
$$

where $x \in \mathfrak{l}, x^{\prime} \in \mathfrak{l}^{\prime}$.

To define a Hermitian form $\{\cdot, \cdot\}$ on $\Lambda_{r e l}^{\frac{\infty}{2}+*}(\tilde{\mathfrak{g}})$, we set $\left\{\mathbf{1}_{r e l}, \mathbf{1}_{r e l}\right\}=1$. We fix a basis $\left\{v_{i}\right\}$ of $\mathfrak{g}$ so that $\left\langle v_{i}, v_{j}\right\rangle=\delta_{i, j}$. Let $u$ run through this basis. We define an antilinear automorphism $\sigma$ of $\tilde{\mathfrak{g}}$ as follows:

$$
\sigma\left(u_{n}\right)=i u_{-n}
$$

Correspondingly,

$$
\sigma\left(u_{n}^{\prime}\right)=-i u_{-n}^{\prime} .
$$

We introduce a Hermitian form on $\Lambda_{\text {rel }}^{\frac{\infty}{2}+*}(\tilde{\mathfrak{g}})$ so that the relations $(6.18)$, where

$$
x \in \tilde{\mathfrak{g}}_{n}, x^{\prime} \in \tilde{\mathfrak{g}}_{n}^{\prime} \text { for } n \neq 0
$$


hold. In the similar way we introduce a Hermitian form on $S^{\frac{\infty}{2}+*}(\tilde{\mathfrak{g}})$, such that

$$
\gamma\left(x^{\prime}\right)^{*}=\gamma\left(\sigma\left(x^{\prime}\right)\right), \quad \beta(x)^{*}=-\beta(\sigma(x))
$$

Then we obtain a Hermitian form $\{\cdot, \cdot\}$ on $W_{r e l}^{\frac{\infty}{2}+*}(\tilde{\mathfrak{g}})$ by tensoring these two forms. It gives a paring: $C^{a, b}(\tilde{\mathfrak{g}}) \longrightarrow C^{b, a}(\tilde{\mathfrak{g}})$. To define a Hermitian form on $C^{a, b}(\tilde{\mathfrak{g}})$, we use the linear map

$$
*: C^{a, b}(\tilde{\mathfrak{g}}) \longrightarrow C^{b, a}(\tilde{\mathfrak{g}})
$$

defined as follows:

$$
\begin{aligned}
& *\left(v \otimes\left(\varepsilon\left(u_{n_{1}}^{\prime}\right) \cdots \varepsilon\left(u_{n_{a}}^{\prime}\right) \tau\left(u_{m_{1}}\right) \cdots \tau\left(u_{m_{b}}\right) \mathbf{1}_{r e l}\right)\right)= \\
& v \otimes\left(\varepsilon\left(u_{-m_{1}}^{\prime}\right) \cdots \varepsilon\left(u_{-m_{b}}^{\prime}\right) \tau\left(u_{-n_{1}}\right) \cdots \tau\left(u_{-n_{a}}\right) \mathbf{1}_{r e l}\right),
\end{aligned}
$$

where $v \in S^{\frac{\infty}{2}+*}(\tilde{\mathfrak{g}}),\left\{n_{i}\right\}_{i=1}^{a}>0$ and $\left\{m_{i}\right\}_{i=1}^{b}<0$. Finally, the Hermitian form on $C^{a, b}(\tilde{\mathfrak{g}})$ is defined by $\left(w_{1}, w_{2}\right)=\left\{i^{a+b} * w_{1}, w_{2}\right\}$ (cf. [FGZ]). We introduce the adjoint operators $d^{*}, d_{c}^{*}$ and the Laplace operator $\triangle=d d^{*}+d^{*} d$.

It was pointed out in [FGZ] that as in the classical theory (see [GH]), there exists an action of $\mathfrak{s l}(2)$ on $H_{\infty}^{*}\left(\mathfrak{l}, \mathfrak{l}_{0}, V\right)$. One can identify $\mathfrak{l}_{n}^{\prime}$ with $\mathfrak{l}_{-n}$ by means of the cocycle $\gamma$. If $\left\{e_{i}\right\}$ is a homogeneous basis in $\mathfrak{l}$, then $\mathfrak{s l}(2)=\langle L, H, \Lambda\rangle$ is defined as follows:

$$
\begin{aligned}
& L=(i / 2) \sum_{m \in \mathbb{Z} \backslash 0} \varepsilon\left(e_{m}\right) \varepsilon\left(e_{m}^{\prime}\right), \\
& H=-\sum_{m \in \mathbb{Z} \backslash 0}: \tau\left(e_{m}\right) \varepsilon\left(e_{m}^{\prime}\right):, \\
& \Lambda=(i / 2) \sum_{m \in \mathbb{Z} \backslash 0} \tau\left(e_{m}\right) \tau\left(e_{m}^{\prime}\right) .
\end{aligned}
$$

We identify $\tilde{\mathfrak{g}}_{n}^{\prime}$ with $\tilde{\mathfrak{g}}_{-n}$ by means of the cocycle $\gamma($ see $(6.11))$, and set

$$
\mathcal{E}=L, \mathcal{H}=H, \mathcal{F}=\Lambda
$$

Then we obtain the $\mathcal{S} \mathcal{L}(2)=\langle\mathcal{E}, \mathcal{H}, \mathcal{F}\rangle$ defined in (5.10). The operators

$$
\left\{\triangle, \mathcal{E}, \mathcal{H}, \mathcal{F}, d, d^{*}, d_{c}, d_{c}^{*}\right\}
$$

are the analogues of the classical operators (6.9). 
Theorem 6.2.- Let $\mathfrak{g}$ be a complex finite-dimensional Lie algebra with a non-degenerate invariant symmetric bilinear form. Then $H^{\frac{\infty}{2}+*}\left(\tilde{\mathfrak{g}}, \tilde{\mathfrak{g}}_{0}, S^{\frac{\infty}{2}+*}(\tilde{\mathfrak{g}})\right)$ is a module over $\hat{D} e r S^{\prime}(2,0)$ with central charge $3 \operatorname{dim} \mathfrak{g}$.

Proof.- By Theorem 5.3, $W_{\text {rel }}^{\frac{\infty}{2}+*}(\tilde{\mathfrak{g}})$ is a module over $\hat{D} e r S^{\prime}(2,0)$ with central charge $3 \operatorname{dim} \mathfrak{g}$. By Corollary 5.1, there is an action of $\hat{S}^{\prime}(2,0)$ on $H^{\frac{\infty}{2}+*}\left(\tilde{\mathfrak{g}}, \tilde{\mathfrak{g}}_{0}, S^{\frac{\infty}{2}+*}(\tilde{\mathfrak{g}})\right)$. We have proved that

$$
\operatorname{Der}_{\text {ext }} S^{\prime}(2,0)=\mathcal{S} \mathcal{L}(2)=\langle\mathcal{E}, \mathcal{H}, \mathcal{F}\rangle
$$

see (5.10). Notice that as in the classical case, the element $\mathcal{F}$ and the differential $d$ do not commute. Nevertheless, there exists an action of $\mathcal{S} \mathcal{L}(2)$ on the relative semi-infinite cohomology according to [FGZ].

Theorem 6.3.- The degree zero part of the $\mathbb{Z}$-grading deg of $S^{\prime}(2,0)$ is isomorphic to the Lie superalgebra of classical operators in Kähler geometry.

Proof.- Recall that the $\mathbb{Z}$-grading $\operatorname{deg}$ on $S^{\prime}(2,0)$ is defined by the element $L_{0} \in W i t t$, see (4.17)-(4.19). One can easily check that

$$
S^{\prime}(2,0)_{\operatorname{deg}=0}=\left\langle L_{0}, E_{-1}, H_{0}, F_{1}, \mathrm{~h}_{0}^{0}, \mathrm{p}_{0}, \mathrm{x}_{-1}, \mathrm{y}_{1}^{0}\right\rangle
$$

The isomorphism of Lie superalgebras

$$
\psi: \mathcal{S} \longrightarrow S^{\prime}(2,0)_{\operatorname{deg}=0}
$$

is given as follows:

$$
\begin{aligned}
& \psi(\triangle)=L_{0}, \psi(L)=E_{-1}, \psi(H)=H_{0}, \psi(\Lambda)=F_{1} \\
& \psi(d)=\mathrm{h}_{0}^{0}, \psi\left(d^{*}\right)=-\mathrm{p}_{0}, \psi\left(d_{c}\right)=x_{-1}, \psi\left(d_{c}^{*}\right)=y_{1}^{0}
\end{aligned}
$$

Corollary 6.1.- The action of $S^{\prime}(2,0)_{d e g=0}$ defines a set of quadratic operators on $W_{\text {rel }}^{\frac{\infty}{2}+*}(\tilde{\mathfrak{g}})$ (correspondingly, on $\left.H^{\frac{\infty}{2}+*}\left(\tilde{\mathfrak{g}}, \tilde{\mathfrak{g}}_{0}, S^{\frac{\infty}{2}+*}(\tilde{\mathfrak{g}})\right)\right)$, which are analogues of the classical ones, and include the semi-infinite Koszul differential $h=h_{0}^{0}$ and the semi-infinite homotopy operator $p_{0}$.

Remark 6.1.- In this work we have realized superconformal algebras by means of quadratic expansions on the generators of the Heisenberg and Clifford algebras related to $\tilde{\mathfrak{g}}$. Note that the differentials on a semi-infinite Weil complex are represented by cubic 
expansions. One can possibly define an additional (to the already known) action of the $N=2 \mathrm{SCA}$ on $W^{\frac{\infty}{2}+*}(\tilde{\mathfrak{g}})$, considering Fourier components of the differentials $d$ and $d^{*}$, $[\mathrm{Fe}]$.

Acknowledgements.- This work has been partly done at the Max-Planck-Institut für Mathematik in Bonn, L'Institut des Hautes Études Scientifiques in Bures-sur-Yvette, and the Institute for Advanced Study in Princeton. I wish to thank MPI, IHES, and IAS for their hospitality and support. I am grateful to B. Feigin, A. Givental, M. Kontsevich, V. Serganova, and V. Schechtman for very useful discussions.

\section{BIBLIOGRAPHY}

[Ad] M. Ademollo, L. Brink, A. D'Adda, R. D'Auria, E. Napolitano, S. Sciuto, E. Del Giudice, P. Di Vecchia, S. Ferrara, F. Gliozzi, R. Musto and R. Rettorino, Dual strings with $U(1)$ colour symmetry, Nucl. Phys. B111 (1976), 77-110.

[Ak] F. Akman, Some cohomology operators in 2-D field theory, Proceedings of the conference on Quantum topology (Manhattan, KS, 1993), World Sci. Publ, River Edge, NJ (1994), 1-19.

[Fe] B. L. Feigin, Private communication.

[Fu] D. B. Fuks, Cohomology of infinite-dimensional Lie algebras, Consultants Bureau, New York and London, 1986.

[FF] B. Feigin, E. Frenkel, Semi-infinite Weil Complex and the Virasoro Algebra, Commun. Math. Phys. 137 (1991), 617-639. Erratum: Commun. Math. Phys. 147 (1992), 647648.

[FGZ] I. Frenkel, H. Garland, G. Zuckerman, Semi-infinite cohomology and string theory, Proc. Natl. Acad. Sci. U.S.A. 83 (1986), 8442-8446.

[FST] B. L. Feigin, A. M. Semikhatov, I. Yu. Tipunin, Equivalence between chain categories of representations of affine $\mathfrak{s l}(2)$ and $N=2$ superconformal algebras, J. Math. Phys. 39 (1998), no 7, 3865-3905.

[G] E. Getzler, Two-dimensional topological gravity and equivariant cohomology, Commun. Math. Phys. 163 (1994) no 3, 473-489.

[GH] P. Griffiths, J. Harris, Principles of algebraic geometry, Wiley-Interscience Publ., New York, 1978.

[KL] V. G. Kac, J. W. van de Leur, On Classification of Superconformal Algebras, in S. J. 
Gates et al., editors, Strings-88, World Scientific 1989, 77-106.

[P1] E. Poletaeva, Semi-infinite Weil complex and $N=2$ superconformal algebra I, preprint MPI 97-78, Semi-infinite Weil complex and superconformal algebras II, preprint MPI 97-79.

[P2] E. Poletaeva, Superconformal algebras and Lie superalgebras of the Hodge theory, preprint MPI 99-136.

[P3] E. Poletaeva, Semi-infinite cohomology and superconformal algebras, Comptes Rendus de l'Académie des Sciences, t. 326, Série I (1998), 533-538.

Elena Poletaeva

Centre for Mathematical Sciences

Mathematics, Lund University

Box 118, S-221 00 Lund, Sweden

elena@maths.lth.se 